\title{
REFRACTIVE INDEX WITHIN THE LENS OF A GOLDFISH EYE DETERMINED FROM THE PATHS OF THIN LASER BEAMS
}

\author{
D. AXelrod, ${ }^{1}$ D. Lerner ${ }^{1}$ and P. J. SANDS ${ }^{2, *}$ \\ 'Biophysics Research Division and Department of Physics, University of Michigan, \\ 2200 Bonisteel Boulevard, Ann Arbor, MI 48109, U.S.A. and ${ }^{2}$ CSIRO Division of Forest Research, \\ P.O. Box 4008, Queen Victoria Terrace, Canberra, ACT 2600, Australia
}

(Received 28 October 1986)

\begin{abstract}
The paths of $15 \mu \mathrm{m}$ diameter laser beams traversing goldfish eye lenses were photographed. Measurements of these photographs gave experimental data for the distance of the exit point of each ray from the lens axis as a function of the corresponding entrance distance. A number of mathematical models with distinct distributions of refractive index within the lens were analysed by tracing rays to simulate the experimental data. The only distributions for which the simulated and experimental data were in agreement have a refractive index $N$ which varies continuously with distance $r$ from the lens center in a manner consistent with that originally proposed by Matthiessen: $N^{2}=a-b r^{2}$. Estimates for the central (1.55-1.57) and surface (1.35-1.38) refractive indices of the goldfish eye lens are derived from the preferred model, but these differ from those previously given by Matthiessen for other species. The optical performance of the lens models is also compared by third-order analyses.
\end{abstract}

Refractive index distribution Goldfish lens Lens models

\section{INTRODUCTION}

It is generally accepted that the refractive index in the lenses of the eyes of the higher animals varies from the lens center to the lens surface although the exact form of this variance is a matter of debate (Fagerholm et al., 1981; Fernald and Wright, 1983; Campbell and Sands, 1984). Measurement of this index distribution is difficult and is generally based on destructive sampling. The refractive index of a sample may be either measured directly by interferometric techniques (Nakao et al., 1968), or indirectly by inference from measurements of the protein concentration within the sample (Philipson, 1969).

Non-destructive techniques for measuring the refractive index within the crystalline lens of necessity yield the index distribution indirectly. For example, Chu (1977), Anderssen and Campbell (1984), and Campbell (1984) describe methods where the exit angles of thin laser beams are measured as a function of their entrance apertures. (The aperture of a ray or beam is the distance from the point of intersection of the ray or beam with the lens to the axis of the lens.) The refractive index distribu-

*To whom correspondence should be addressed. tion is then derived via a mathematical process called an Abel integral inversion. This method assumes that the distribution has a certain mathematical form and yields numerical estimates of any parameters characterizing this form. It is not easy to adapt this technique to arbitrary distributions.

In this paper we describe the results of applying an alternative non-destructive technique to the measurement of the refractive index distribution within the lens of a goldfish eye. The paths of narrow laser beams traversing a meridional plane of the lens were photographed and the entrance and exit apertures of each ray were measured. A series of distinct mathematical models of the refractive index distribution within the lens were postulated. Ray-trace analysis of these models then generated data which were compared with the experimental data.

\section{EXPERIMENTAL}

A lens was dissected from a goldfish eye and placed in a commercial $1 \mathrm{~cm} \times 5 \mathrm{~cm}$ glass cuvette with four glass faces (designed for fluorescence spectroscopy) which had been filled with buffered saline solution at physiological ionic strength. The refractive index of this solution was $N_{\mathrm{a}}=1.334$. The top of the cuvette was 
sealed with parafilm. The sealed cuvette was placed on its side on a fixed upright microscope stage (American Optical 110) such that the goldfish lens (called "the sample") was in the center of the field of view.

A well-defined ray of light from a $0.5 \mathrm{~mW}$ helium-neon laser was directed towards the sample perpendicularly to the optical axis of the microscope. Before incidence upon the sample the ray passed through a $2 \mathrm{~mm}$ focal length glass lens positioned so that the sample was at its focal point. This focusing lens reduced the laser beam's diameter from about $1 \mathrm{~mm}$ to about $15 \mu \mathrm{m}$ while preserving its approximate collimation as it passed through the sample. The height of the beam was adjusted so that it propagated in a plane parallel to the microscope stage and halfway between the near and far surfaces of the lens. Assuming the lens to be a sphere (see below), this plane is a meridional plane of the sample and will be referred to as "the meridional plane".

The sample was observed through the microscope fitted with a $4 \times$ objective such that the meridional plane (in which the laser beam propagates) was in focus. Scattering of light in the sample and Rayleigh scattering in the buffer solution marked the apparent path of the beam through the goldfish lens. This was photographed with a microscope mounted $35 \mathrm{~mm}$ camera. Although the apparant path was distorted by the bulk of the sample, the positions of entrance and exit points were observed without distortion.

A total of 124 photographs were taken with randomly selected but closely spaced entrance apertures. These were obtained by adjusting the lens position transverse to the incident beam with the microscope stage translator. A typical photograph is shown in Fig. 1. Also shown are the aperatures $y_{1}$ and $y_{2}$ of the entrance and exit points of the ray. These are defined with respect to the optical axis of the goldfish lens parallel to the incident laser beams. The data were not all gathered from the same lens: as each lens suffered from surface cloudiness after about $20 \mathrm{~min}$ in the buffer solution, the data are a combination of measurements taken on 9 separate lenses from the same strain of fish, but their ages and sex were not recorded. The cause of surface cloudiness was not known, but there was no change in refractive properties over time.

The photographs allowed us to determine that the goldfish lenses were spherical. The greatest departure from sphericity corresponded

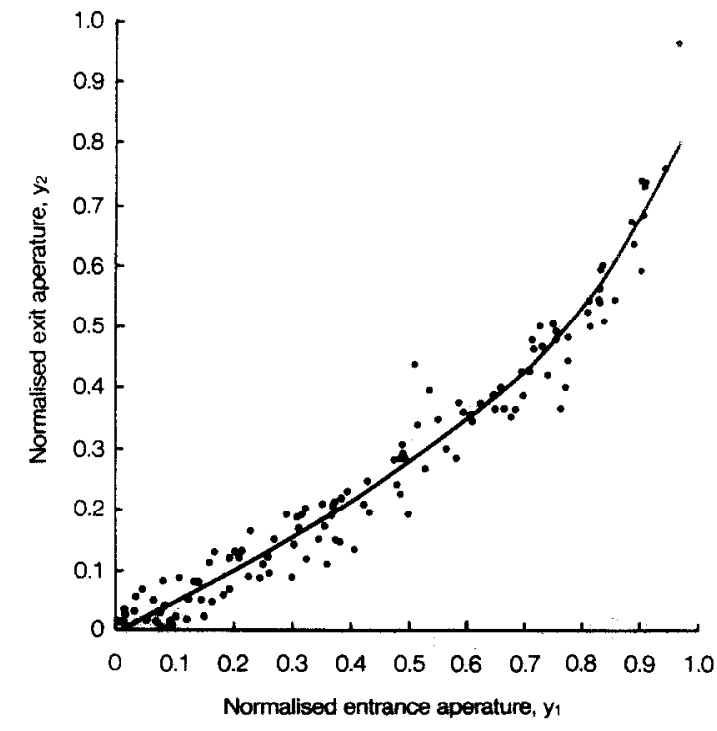

Fig. 2. The dots (O) are the observed values of the exit aperture $y_{2}$ of thin laser beams traversing a goldfish lens plotted as a function of the entrance aperture $y_{1}$ of the beam, both normalized by dividing by the radius of the lens. The solid line (-) is the simulated data obtained by ray tracing a model spherical lens in which the refractive index at a distance $r$ from the center of the lens is given by $N(r)^{2}=N_{\mathrm{c}}^{2}+\left(N_{\mathrm{s}}^{2}-N_{\mathrm{c}}^{2}\right)(r / R)^{2}$ where $R$ is the radius of the lens, $N_{\mathrm{s}}=1.36$ is the refractive index at the surface of the lens, and $N_{\mathrm{c}}=1.555$ is the refractive index at the center of the lens.

to a $1 \%$ difference in polar and equatorial diameter consistent with observations of Sadler (1973). The mean diameter of the 9 lenses upon which measurements were based was $2.2 \pm$ $0.2 \mathrm{~mm}$. It was also assumed that the refractive index distribution within the lens was spherical and hence the orientation of the lens was unimportant. The surface and central refractive indices were not measured.

The actual measurements of $y_{1}$ and $y_{2}$ from the photographs were made using a bubble chamber photograph scanner (most commonly used in high energy particle physics). This allowed us to digitize the locations of the entry and exit points of the beam, the location of one arbitrary point on the incident beam (to define its direction), and the location of an arbitrary point on the circumference of the goldfish lens (to define its centre). The digitized coordinates of these four points were then used to compute $y_{1}$ and $y_{2}$. In order to correct for slight variations in the diameters of the 9 lenses used, $y_{1}$ and $y_{2}$ were expressed as a fraction of the radius of the lens. These normalized values of the observed $y_{1}$ and $y_{2}$ are plotted in Fig. 2 . 

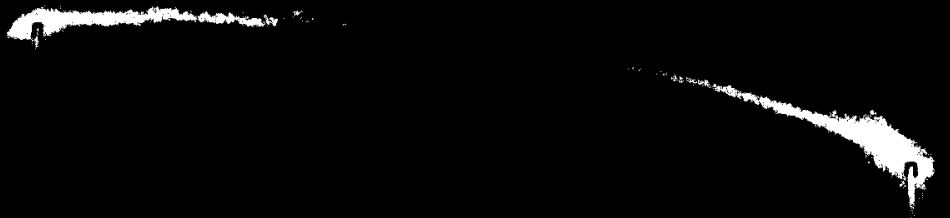

Fig. 1. Photograph of a laser beam traversing a goldfish lens. The refraction of the beam at each surface and the curvature of its path within the lens are clearly visible. The entrance and exit apertures $y_{1}$ and $y_{2}$ of the beam are indicated. 


\section{THEORETICAL METHODS}

Mathematical models of a unit-radius goldfish lens were constructed with various distinct families of refractive index distribution in the lens characterized by two or more parameters. Each model was analyzed by assigning plausible values to each of its parameters, and then tracing meridional rays at various apertures $y_{1}$ and recording the corresponding exit aperture $y_{2}$. This gave a simulated set of data corresponding to the observed data of Fig. 2.

The numerical values of the various parameters of each model were systematically adjusted and the model re-analyzed until the simulated relationship between $y_{2}$ and $y_{1}$ best agreed with the observed data in Fig. 2. The solid curve in Fig. 2 is the best fit obtained for any model examined: the simple spherical model described below.

All ray tracing was performed numerically using the computer program Drishti written by one of us (Sands, 1984). Drishti is a program for analyzing symmetrical optical systems incorporating inhomogeneous media and had been used to conduct a systematic study of image formation by the rat eye (Campbell, 1988; Sands, 1988, 1984).

\section{ANALYSIS OF GOLDFISH LENS-MODELS}

Guidelines for the choice of refractive index distributions

The choice of the refractive index distributions used in the lens models was guided by general observations from a consideration of the processes of refraction of light within a medium with a continuous refractive index, or at a surface separating media with distinct refractive indices. These observations are independent of any assumption of spherical symmetry and are:

(a) If the refractive index within the lens is homogeneous, the graph of $y_{2}$ as a function of $y_{1}$ is continuous and concave downwards.

(b) If the refractive index within the lens has a discontinuity, then so does the graph of $y_{2}$ as a function of $y_{1}$, and the bigger the discontinuity in refractive index, the bigger the discontinuity in $y_{2}$.

(c) If the refractive index within the lens is continuous but its gradient is discontinuous, then $y_{2}$ is a continuous function of $y_{1}$ but its slope is discontinuous, and the bigger the discontinuity in gradient the bigger the discontinuity in slope. (d) If the refractive index distribution is both continuous and smooth, then the graph of $y_{2}$ as a function of $y_{1}$ is also continuous and smooth.

Accordingly the observed data of Fig. 2 support the assumption that the refractive index distribution within the goldfish lens is both continuous and smooth, or that any discontinuities in the refractive index or its gradient are either small or are confined to the extreme outer regions of the lens.

\section{Refractive index near the lens surface}

If the lens has a homogeneous outer shell it can be shown by applying Snell's law and elementary geometry that the normalized exit and entrance apertures are related by

$$
\begin{aligned}
y_{2}=2 n y_{1} \sqrt{1-n^{2} y_{1}^{2}} \sqrt{1-y_{1}^{2}} & \\
& +\left(2 n^{2} y_{1}^{2}-1\right) y_{1}
\end{aligned}
$$

where $n=N_{\mathrm{a}} / N_{\mathrm{s}}$ and $N_{\mathrm{s}}$ is the refractive index of the shell and $N_{\mathrm{a}}$ that of the medium in which the lens is immersed, provided

$$
y_{1} \geqslant r_{\mathrm{c}} N_{\mathrm{s}} / R N_{\mathrm{a}}
$$

where $R$ is the lens radius and $r_{\mathrm{c}}$ the inner radius of the shell. The condition (2) guarantees that the rays propagate only in the homogeneous shell. Equation (1) can be inverted to express $N_{s}$ in terms of $N_{\mathrm{a}}, y_{1}$ and $y_{2}$

$$
\begin{aligned}
N_{s}^{2}=2 N_{\mathrm{a}}^{2} y_{1}^{2} / & \left\{\left(1+y_{1} y_{2}\right)\right. \\
\times & {\left.\left[1 \pm \sqrt{1-\left(\frac{y_{1}+y_{2}}{1+y_{1} y_{2}}\right)^{2}}\right]\right\} . }
\end{aligned}
$$

We used these results to explore the possibility that the lens has an homogeneous outer shell.

For instance, if the ray with $y_{1}=0.95$, $y_{2}=0.78$ (Fig. 2) passes through a homogeneous shell, then equations (2) and (3) imply that $N_{\mathrm{s}}=1.4416, r_{\mathrm{c}} \leqslant 0.88 R$. This refractive index is much higher than values reported elsewhere, e.g. 1.366 or 1.386 (Matthiessen, 1880). Now suppose the shell is so thin that only rays with $y_{1}$ close to 1 transverse it. Extrapolation of the observed data of Fig. 2 suggest $0.9 \leqslant y_{2} \leqslant 0.95$, and equations (2) and (3) give $1.35 \leqslant N_{\mathrm{s}} \leqslant 1.37,0.975 R \leqslant r_{\mathrm{c}} \leqslant 0.987 R$. These indices are consistent with those of Matthiessen, and the homogeneous shell is indeed quite thin and can be ignored.

\section{A simple spherical inhomogeneous lens model}

The first lens model we analyzed had a spherical lens and a refractive index distribution 
in which the surfaces of constant index are concentric spheres. The refractive index at a distance $r$ from the lens centre is given by

$$
N(r)^{2}=N_{\mathrm{c}}^{2}+\left(N_{\mathrm{s}}^{2}-N_{\mathrm{c}}^{2}\right)(r / R)^{2}
$$

where $R$ is the lens radius, and $N_{\mathrm{s}}$ and $N_{\mathrm{c}}$ the surface and central refractive indices, respectively. This model was suggested by those of Campbell and Sands (1984) and Matthiessen (1880). Because of the normalization of the observed values of $y_{1}$ and $y_{2}$ to a lens of unit radius, we assumed $R=1$.

The selection of values for the parameters $N_{\mathrm{s}}$ and $N_{\mathrm{c}}$ was helped by the existence of a simple expression for the slope $y_{p}$ of the functional relationship between $y_{2}$ and $y_{1}$ for paraxial rays (i.e. for small $y_{1}$ ) and for the paraxial focal length $f$ of the lens. If the lens is immersed in a medium of index $N_{\mathrm{a}}$, then

$$
\begin{aligned}
y_{\mathrm{p}} & =2 N_{\mathrm{a}} N_{\mathrm{s}} / N_{\mathrm{c}}^{2}-1 \\
f & =R /\left(1-y_{\mathrm{p}}\right) .
\end{aligned}
$$

These equations can be derived by solving the differential equations for rays in a spherically symmetric index distribution (e.g. Marchand, 1978) and then taking the paraxial limit. The second of equation (5) is valid for any spherically symmetric lens model.

When the parameters of the model were being estimated we used equation (5) to choose combinations of $N_{\mathrm{s}}$ and $N_{\mathrm{c}}$ so that the values of $y_{\mathrm{p}}$ for the simulated and observed data sets were in agreement (from Fig. $2 y_{\mathrm{p}}=0.45-0.55$, $f \approx 1.82 R-2.22 R$ ). The free parameter was then varied in an attempt to obtain consistency between the two data sets for large apertures. For example, if $N_{\mathrm{s}}=1.36$ and $y_{\mathrm{p}}=0.5$, equation (5) gives a central index of $N_{\mathrm{c}}=1.555$.

This lens model was analyzed by ray tracing and the solid curve of Fig. 2 is a plot of the data obtained for this model. The agreement between the model and the observations is striking and the choice $N_{\mathrm{s}}=1.36, N_{\mathrm{c}}=1.555$ is typical of the best fits obtained with any model. Because of the scatter in the observed data a range of values of $N_{\mathrm{s}}$ and $N_{\mathrm{c}}$ were found for which an equivalent fit to the observed data was obtained, e.g. $N_{\mathrm{s}}=1.35-1.38, N_{\mathrm{c}}=1.55-1.57$.

\section{Spherical polynomial inhomogeneous model}

Our experimental technique did not give reliable values for the exit aperture $y_{2}$ of rays with an entrance aperture close to 1 . It was therefore of interest to examine the consequences of modifications to equation (4) close to the lens surface. Accordingly, we examined a spherical model with the polynomial index distribution

$$
N(r)^{2}=N_{\mathrm{c}}^{2}+\left(N_{\mathrm{s}}^{2}-N_{\mathrm{c}}^{2}\right)(r / R)^{2}+n_{4}(r / R)^{4}
$$

where $n_{4}$ is a new free parameter. We varied $n_{4}$ (with $N_{\mathrm{s}}=1.36, N_{\mathrm{c}}=1.555$ ) from -0.07 to 0.0825 , corresponding to lens surface indices in the range 1.334-1.39. However, ray tracing showed that the fit to the observed data did not differ significantly from that shown in Fig. 2 for the simple model, i.e. the case $n_{4}=0$.

\section{Spheroidal inhomogeneous model}

We studied the importance of spherical symmetry by considering a model in which the lens was a prolate spheroid with the optical axis as its axis of rotation, and in which the refractive index distribution was spheroidal (Sands, 1984) with surfaces of constant index having the same shape as the lens surface. For such distribution the refractive index at the point $(x, y, z)$ is

$$
\begin{aligned}
N(x, y, z)^{2}= & N_{\mathrm{c}}^{2}+\left(N_{\mathrm{s}}^{2}-N_{\mathrm{c}}^{2}\right) \\
& \times\left[\left(\boldsymbol{R}_{y} / \boldsymbol{R}_{x}\right)^{2} x^{2}+y^{2}+z^{2}\right] / \boldsymbol{R}_{y}^{2}
\end{aligned}
$$

where the origin is at the lens center. $N_{\mathrm{s}}$ and $N_{\mathrm{c}}$ are the refractive indices at the surface and center of the lens, and $R_{x}$ and $R_{y}$ are the semi-axes of the lens surface along and transverse to the optical axis, respectively.

We considered models with different lens shapes as determined by $\left(R_{x} / R_{y}\right)$. We set $N_{\mathrm{s}}=1.36$ and varied $N_{\mathrm{c}}$ until the simulated data had $y_{\mathrm{p}}=0.5$. Increasing $\boldsymbol{R}_{x} / \boldsymbol{R}_{y}$ only slightly changed the quality of the fit of the observed values of $y_{2}$, but reduced the $N_{\mathrm{c}}$ required for agreement between observed and simulated data. For example, if $R_{x} / R_{y}=1.1$ the central index was reduced to 1.515 . In our case $R_{x} / R_{y}<1.02$ and the central indices must be high.

Lens model with an inhomogeneous core and an homogeneous shell

We examined models in which the lens had an inhomogeneous spherical core surrounded by an homogeneous shell. An example had

$$
N(r)^{2}=\left\{\begin{array}{lr}
N_{\mathrm{s}}^{2} & \left(R \geqslant r \geqslant r_{\mathrm{c}}\right) \\
N_{\mathrm{c}}^{2}+\left(N_{\mathrm{s}}^{2}-N_{\mathrm{c}}^{2}\right)\left(r / r_{\mathrm{c}}\right)^{2} & \left(r_{\mathrm{c}} \geqslant r \geqslant 0\right)
\end{array}\right.
$$

where $N_{\mathrm{c}}$ and $N_{\mathrm{s}}$ are the refractive indices at the lens center and surface, $r_{\mathrm{c}}$ the radius of the core and $R$ the lens radius. We found that the 
agreement between the simulated and observed data was simultaneously possible for both large and small $y_{1}$ only if the shell thickness was essentially zero. This confirms the discussion following equation (3).

Lens models with an homogeneous core and an inhomogeneous shell

The final class of lens model we examined was lenses with an homogeneous core surrounded by an inhomogeneous shell. The refractive index in the shell was assumed to have a continuous distribution and a smooth gradient. An example had

$$
\begin{aligned}
& N(r)^{2}= \\
& \begin{cases}N_{\mathrm{c}}^{2} & \left(0 \leqslant r \leqslant r_{\mathrm{c}}\right) \\
N_{\mathrm{c}}^{2}+\left(N_{\mathrm{s}}^{2}-N_{\mathrm{c}}^{2}\right)\left(\frac{r-r_{\mathrm{c}}}{R-r_{\mathrm{c}}}\right)^{2} & \left(r_{\mathrm{c}} \leqslant r \leqslant R\right)\end{cases}
\end{aligned}
$$

with the same notation as above. This model was suggested by that proposed for the teleost lens by Fernald and Wright (1983), and by measurements of the protein concentration in the human lens (Fagerholm et al., 1981). We analyzed models with various core sizes and found that the smaller the core, the better the simulated and observed data sets agreed. This model overestimates $y_{2}$ for small $y_{1}$ and underestimates $y_{2}$ for large $y_{1}$. These results are illustrated in Fig. 3. Very small core sizes are indicated (e.g. $r_{c}<0.2 R$ ).

Comparison of third-order spherical aberration for the lens models

The program Drishti which we used to analyze the various models computes the thirdorder or Seidel aberration coefficients (Sands, 1984). Because of the spherical symmetry of the systems, the quality of the image is determined by spherical aberration. Table 1 lists the values of the coefficient $\sigma_{1}$ of third-order spherical aberration for the various lens models we considered.

For comparison, a homogeneous spherical

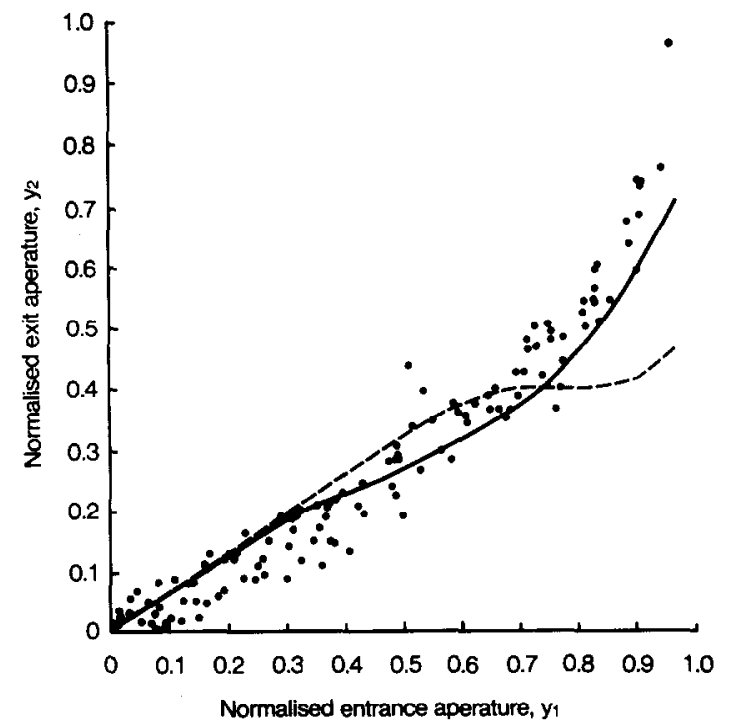

Fig. 3. The dots (O) are the observed data. The solid line $(\longrightarrow)$ is the simulated exit-aperture relationship for a lens with an homogeneous spherical core surrounded by an inhomogeneous shell; see equation (9). The radius of the core is $r_{\mathrm{c}}=0.3 R$, and the refractive indices of the lens core and at the lens surface are $N_{\mathrm{c}}=1.56$ and $N_{\mathrm{s}}=1.36$. The dashed curve (----) is the simulated data for the same model but with a core radius $r_{\mathrm{c}}=0.6 R$.

lens of refractive index 1.779 (for which $y_{p}=0.5$ ) has been included. The model with an inhomogeneous shell [equation (9)] is even more undercorrected for spherical aberration than the homogeneous lens. The other models, all of whose index distributions are approximated by equation (4), are slightly overcorrected for spherical aberration, but give much better imagery than would a homogeneous lens.

The coefficient $\sigma_{1}$ for model lenses with index distributions given by equation (4) is relatively insensitive to the central and surface indices. For example, if $N_{\mathrm{c}}$ and $N_{\mathrm{s}}$ are chosen so that the focal length of the lens varies over the range 2.05-2.75R, $\sigma_{1}$ varies between 0.08 and 0.11 . High indices reduce $\sigma_{1}$.

\section{DISCUSSION}

We have analyzed a number of distinct refractive index distributions as possible models for

Table 1. Coefficient of third-order spherical aberration for the various lens models

\begin{tabular}{|c|c|c|}
\hline Equation No. & Model & $\sigma_{1}$ \\
\hline $\begin{array}{l}4 \\
6\end{array}$ & $\begin{array}{l}\text { Simple spherical inhomogeneous, } N_{\mathrm{s}}=1.36, N_{\mathrm{c}}=1.555 \\
\text { Spherical polynomial inhomogeneous, } \begin{array}{l}n_{4}=0.0825 \\
n_{4}=-0.07\end{array}\end{array}$ & $\begin{array}{l}0.096 \\
0.17 \\
0.028\end{array}$ \\
\hline 7 & Spheroidal inhomogeneous & 0.12 \\
\hline $\begin{array}{r}8 \\
9 \\
-\end{array}$ & $\begin{array}{l}\text { Inhomogeneous core, } r_{\mathrm{c}}=0.88 R, N_{\mathrm{s}}=1.442, N_{\mathrm{c}}=1.578 \\
\text { Inhomogeneous shell, } r_{\mathrm{c}}=0.6 \\
\text { Spherical homogeneous lens, } N=1.779\end{array}$ & $\begin{aligned} & 0.091 \\
- & 0.54 \\
- & 0.34\end{aligned}$ \\
\hline
\end{tabular}
considered 
the distribution within a goldfish lens. The best model was selected on the extent to which the relationship between the exit and entrance apertures determined by tracing rays through the model agrees with the experimentally obtained relationship of Fig. 2. On this basis the simple distribution of refractive index given by equation (4) with neither an homogeneous core nor an homogeneous shell is preferred. The refractive indices $N_{\mathrm{c}}$ and $N_{\mathrm{s}}$ at the lens center and surface must be in the ranges $N_{\mathrm{c}}=1.55-1.57$, $N_{\mathrm{s}}=1.35-1.38$, and the choice $N_{\mathrm{c}}=1.555$, $N_{\mathrm{s}}=1.36$ is probably optimal.

The form of our preferred distribution is consistent with that found by Matthiessen $(1880,1882,1887)$ for a range of species of fish and for the dolphin. However, our preferred central refractive index is higher than the typical values reported by Matthiessen (1.555, cf. $1.50-1.54)$, whilst our surface index is lower than his (1.36, cf. 1.38-1.386). Using our preferred indices the paraxial focal length of the goldfish eye computed from equation (5) is $2 R$, where $R$ is the radius of the lens. Note that focal lengths in the range $1.86 R$ to $2.15 R$ are consistent with the observed data of Fig. 2. The focal length calculated using Matthiessen's refractive indices is in the range $2.3-2.8 R$, and similar values are reported by others, e.g. Sadler (1973). However, Sroczynski (1977) reports focal lengths in the range $2.17-2.29 R$ for roach lenses.

We have attempted to determine a direct measure of the focal length and spherical aberration of the goldfish lens from a random sample of the original photographs. Whereas equation (5) gives the paraxial focal length, these direct measures give the distance $F$ from the lens centre to the point where a ray of finite aperture crosses the optical axis, and are affected by the presence of spherical aberration. For a range of apertures $y_{1}>0.65$, we estimated $F$ to be of the order of $2.14 R$, and increasing with aperture.

Our findings are inconsistent with the conclusion of Fernald and Wright (1983) that the lens of the teleost fish-eye has an homogeneous core of radius $0.67 R$ and refractive index $N_{\mathrm{c}}=1.60$ with an inhomogeneous shell whose surface index is 1.38. Our examination of models with this class of distribution, e.g. equation (9), showed for the goldfish eye that they cannot reproduce our observed exit aperture data, and they are poorly corrected for spherical aberration. Campbell and Sands (1984) also exam- ined the Fernald and Wright (1983) model and concluded (a) that it did not have the high quality imagery reported by Fernald and Wright and (b) that a simple model with a smoothly varying refractive index of the form of equation (4) with $N_{\mathrm{c}}=1.5383$ and $N_{\mathrm{s}}=1.38$, (i) has the required image quality and (ii) agreed with the observed focal ratios of peeled lenses (Fernald and Wright, 1983) if the finite size of their laser beams were taken into account.

An homogeneous core model for the refractive index distribution in the human lens is also implied by the observations of Fagerholm et al. (1981) of the protein distribution within the lens. However, one of us (Sands) has made a preliminary study of a wide-angle model of the human eye using a realistic geometry and a refractive index distribution derived from Fagerholm et al. (1981). Image quality from this model was markedly inferior to that from a model of identical geometry but which lacked an homogeneous core.

Our experimental data lacked a significant number of observations for entrance apertures exceeding $0.9 R$. Such data is essential to judge the validity or otherwise of models with a homogeneous shell, e.g. the model of equation (8). However, we believe the lens has no homogeneous shell, or if it does the shell must be quite thin. For instance, Matthiesson consistently used a surface index in the range 1.38-1.385. Our preferred model has a refractive index of 1.38 at a distance $0.95 R$ from the lens center, suggesting a maximum shell thickness of $0.05 R$. We doubt that our experimental technique could detect such a thin shell as from equation (2) the entrance apertures of rays confined to this shell must exceed $0.98 R$. Moreover, other analyses, e.g. of the model of equation (6), demonstrated that the surface index of the lens could be varied over a wide range without any appreciable affect on the fit of the model to the observed exit aperture data.

These observations emphasize the importance of a bulk measurement of the refractive index at the lens surface. Suppose bulk measurement of refractive indices can be made to an accuracy of 0.01 units. In our preferred model the refractive index increases from 1.36 to 1.37 over a distance of $0.025 R$ from the lens surface, but decreases from 1.555 to 1.545 over a distance of $0.23 R$ from the lens center. Thus bulk measurement would require tiny samples to determine the surface index of our goldfish lens, and would have to be quite accurate to distinguish an 
homogeneous core from the distribution in our model.

Our preferred model was over-corrected for spherical aberration (Table 1). Our estimation of $F$, the lens-centre to axis-crossing distance, suggested that $F$ increases with increasing aperture. This is consistent with an over-corrected lens. However, Sivak and Kreuzer (1983) showed that spherical aberration in three fish species, including the goldfish, was undercorrected and Sroczynski (1977) showed that spherical aberration in the roach was undercorrected except for lenses with focal length $f=2.16 R$ which were slightly over-corrected. We cannot explain this inconsistency. If the exit angles of the laser beams had been available, we would have been able to directly measure spherical aberration.

There are many other smooth functions we could have used to represent the refractive index distribution in the lens. However, on the basis of those we considered, we claim that the function $N(r)$ representing the index distribution must be concave downwards or, if not, the point of inflexion must be far from the lens center, and that the refractive power of the distribution in some sense must be uniformly distributed throughout the lens. The second requirement eliminates homogeneous regions and helps reduce spherical aberration.

\section{CONCLUSIONS}

We have demonstrated that the use of thin laser beams is a practical method to simulate the paths of rays through the fisheye lens, and that the refractive index distribution within the lens can be inferred from data relating exit aperture to entrance aperture. We strongly recommend when the technique described in this paper is used that the directions of the laser beams after exiting from the lens be recorded. This requires that one extra point for each beam be digitized. The additional information provided by the exit angles would help resolve ambiguities in the choice of models and would enable spherical aberration to be determined experimentally.

Using our method we concluded that the refractive index distribution within a fisheye lens can be described by the simple model of equation (4) and does not have an homogeneous shell or an homogeneous core. This distribution readily reproduces the exit aperture relationship observed for the goldfish lens, is consistent with the observations of Matthiesson (1880, 1882, 1887), and results in images in which spherical aberration is low, although the resulting spherical aberration is inconsistent with that discerned by Sivak and Kreuzer (1983).

Acknowledgements-Supported in part by USPHS No. 14565 (to D.A.). The assistance of W. S. Jagger for access to his unpublished translation of Matthiessen's work is acknowledged. We also thank Dr W. C. Parkinson for the use of his bubble chamber photo scanner, and Dr S. Easter for his original suggestion to study this problem and his numerous helpful comments. Our thanks are also due to Anita Gracie for typing this manuscript.

\section{REFERENCES}

Anderssen R. S. and Campbell M. C. W. (1984) Computational aspects associated with the direct use of indirect measurements: refractive index of biological lenses. Proc. Comp. Tech. and Appl. Conf. (CTAC-83), Sydney University (Edited by Noyle J. and Fletcher C. A. J.). North-Holland, Amsterdam (1983).

Campbell M. C. W. (1984) Measurement of refractive index in an intact crystalline lens. Vision Res. 24, 409-515.

Campbell M. C. W. and Sands P. J. (1984) Optical quality during crystalline lens growth. Nature, Lond. 312, 5991, pp. 291-292.

Camphell M. C. W. (1988) A full-field gradient refractive index eye model. In Modelling the Eye with Gradient Index Optics (Edited by Hughes A.). Cambridge Univ. Press.

Chu P. L. (1977) Nondestructive measurement of index profile of an optical fibre preform. Elec. Lett. 13, 736-738.

Fagerholm P., Philipson B. T. and Lindström B. (1981) Normal human lens-the distribution of protein. Expl Eye Res. 33, 615-620.

Fernald R. D. and Wright S. E. (1983) Maintenance of optical quality during crystalline lens growth. Nature, Lond. 301, 618-620.

Marchand E. W. (1978) Gradient Index Optics, p. 166 Academic Press, New York.

Matthiessen L. (1880) Untersuchungen ueber den Aplanatismus und die Periscopie der Kerystallinse in den Augen der Fische. Pfleugers Arch. 21, 287-307.

Matthiessen L. (1882) Ueber die Beziehungen, welche zwischen dem Brechungsindex des Kerncentrums der Kristallinse und den Dimensionen des Auges bestehen. Pfleugers Arch. 27, 510-523.

Matthiessen L. (1887) Beitraege zur Dioptrik der Krystallinse. Z. vergl. Augenheilk. 1, 7-126.

Nakao S., Fumimoto S., Nagata R. and Iwata K. (1968) Model of refractive-index distribution in the rabbit crystalline lens. J. opt. Soc. Am. 58, 1125-1130.

Philipson B. (1969) Distribution of protein within the normal rat lens. Invest. Ophthal. 8, 260-269.

Sadler J. D. (1973) The focal length of the fish eye lens and visual acuity. Vision Res. 13, 417-423.

Sands P. J. (1984) Drishti, a computer program for analysing symmetrical optical systems incorporating inhomogeneous media. Tech. Rept. 8, CSIRO Division of Computing Research, Canberra, Australia.

Sands P. J. (1988) Modelling the geometrical optics of eyes. In Modelling the Eye With Gradient Index Optics (Edited by Hughes A.). Cambridge Univ. Press.

Sivak J. G. and Kreuzer R. O. (1983) Spherical aberration of the crystalline lens. Vision Res. 23, 59-70.

Sroczynski S. (1977) Spherical aberration of crystalline lens in the roach, Rutilus rutilus L. J. comp. Physiol. 121, 135-144. 